\title{
2MASS J15460752-6258042: a mid-M dwarf hosting a prolonged accretion disc
}

\author{
Jinhee Lee, ${ }^{1,2}$ Inseok Song ${ }^{2 \star}$ and Simon Murphy ${ }^{3}$ \\ ${ }^{1}$ School of Space Research, Kyung Hee University, 1732, Deogyeong-Daero, Giheung-gu, Yongin-shi, Gyunggi-do 17104, Korea \\ ${ }^{2}$ Department of Physics and Astronomy, The University of Georgia, Athens, GA 30602, USA \\ ${ }^{3}$ School of Science, University of New South Wales Canberra, ACT 2611, Australia
}

Accepted XXX. Received YYY; in original form ZZZ

\begin{abstract}
We report the discovery of the oldest ( $55 \mathrm{Myr})$ mid-M type star known to host on-going accretion. 2MASS J15460752-6258042 (2M1546, spectral type M5, 59.2 pc) shows spectroscopic signs of accretion such as strong $\mathrm{H} \alpha$, He I, and [O I] emission lines, from which we estimate an accretion rate of $\sim 10^{-10} \mathrm{M}_{\odot} \mathrm{yr}^{-1}$. Considering the clearly detected infrared excess in all WISE bands, the shape of its spectral energy distribution and its age, we believe the star is surrounded by a transitional disc, clearly with some gas still present at inner radii. The position and kinematics of the star from Gaia DR2 and our own radial velocity measurements suggest membership in the nearby 55 Myr-old Argus moving group. At only 59pc from Earth, 2M1546 is one of the nearest accreting mid-M dwarfs, making it an ideal target for studying the upper limit on the lifetimes of gas-rich discs around low mass stars.
\end{abstract}

Key words: accretion, accretion discs - stars: late-type - stars: pre-main-sequence - open clusters and associations: individual

\section{INTRODUCTION}

Stars inevitably harbour discs in the early stages of the star formation process and the lifetime of such discs is one of the most important parameters for understanding early stellar evolution. Moreover, because gas giant planet formation must occur while the disc is gas-rich, the disc lifetime is also a crucial parameter in planet formation scenarios. By surveying young (2-30 Myr) clusters, Haisch et al. (2001) showed the lifetime of gas-rich discs is $\sim 2 \mathrm{Myr}$ and ongoing accretion is rare beyond ages of $\sim 10$ Myr. Mamajek (2009) noted that the disc dissipation timescale should be dependent on the mass and hence spectral type (temperature) of the host star, and Ribas et al. (2015) showed that highmass $\left(>2 \mathrm{M}_{\odot}\right)$ stars dispersed their discs up to twice as fast as lower mass stars.

Recently, several examples of circumstellar disc accretion at ages greater than 10 Myr have been identified (e.g., PDS 66- 20 Myr; Mamajek et al. 2002, HD 21197- 30 Myr; Moór et al. 2011, 49 Ceti- 40 Myr; Zuckerman \& Song 2012, WISE J080822.18-644357.3- 45 Myr; Silverberg et al. 2016; Murphy et al. 2018, J0446A \& B, J0949A \& B; Silverberg et al. 2020), and they have been treated as unusual anomalies without delving into the problem of prolonged gas accretion at extreme ages. In spite of the small number of cases, such

^ E-mail: song@uga.edu old pre-MS stars hosting accretion discs can be challenging to the hypothesis for the rapid planet formation (Pfalzner \& Bannister 2019; Manara et al. 2018; Najita \& Kenyon 2014; Greaves \& Rice 2010) and provide the upper limit on the life times of gas-rich discs.

The young M5 star 2MASS J15460752-6258042 (hereafter 2M1546) was serendipitously found by us in a survey of low-mass moving group candidate members from Gaia DR2 (Lee et al. in preparation). 2M1546 was first observed spectroscopically and classified as a T Tauri star with strong $\mathrm{H} \alpha$ emission by Miszalski \& Mikołajewska (2014) during their survey for new symbiotic stars selected from the AAO/UKST SuperCOSMOS H $\alpha$ Survey. In this work, we analyse optical spectra of 2M1546, its IR excess and spectral energy distribution, and evaluate its age based on the kinematic membership of nearby young moving groups. From this analysis we conclude that $2 \mathrm{M} 1546$ is the oldest (55 Myr) and one of the nearest (59 pc) accreting M-type stars discovered to date.

\section{SPECTROSCOPIC OBSERVATIONS AND DATA ANALYSIS}

We observed 2M1546 using the Wide Field Spectrograph (WiFeS; Dopita et al. 2007) mounted on the ANU 2.3-m telescope at Siding Spring Observatory during June 2018 
Table 1. Summary of ANU 2.3-m/WiFeS observations of 2M1546.

\begin{tabular}{|c|c|c|c|c|c|c|c|c|c|}
\hline \multirow[t]{2}{*}{ UT date } & \multirow[t]{2}{*}{ MJD } & \multirow[t]{2}{*}{ Grating } & \multirow[t]{2}{*}{$\mathrm{SpT}$} & \multirow{2}{*}{$\begin{array}{c}\mathrm{RV} \\
\left(\mathrm{km} \mathrm{s}^{-1}\right)\end{array}$} & \multirow{2}{*}{$\begin{array}{c}v_{10}[\mathrm{H} \alpha] \\
\left(\mathrm{km} \mathrm{s}^{-1}\right)\end{array}$} & \multicolumn{4}{|c|}{ Equivalent width $(\AA)^{\mathrm{a}}$} \\
\hline & & & & & & $\mathrm{H} \alpha$ & He I $\lambda 6678$ & [O I] $\lambda 6300$ & Li I $\lambda 6708$ \\
\hline 2018 Jun 4 & 58273.5813 & R7000 & M5 & $-4.4 \pm 0.9$ & 366 & -210 & -1.6 & -3.6 & 0.43 \\
\hline 2019 Feb 15 & 58529.6854 & R7000 \& B3000 & M5 & -3.5 & 310 & -120 & -0.8 & -3.8 & 0.60 \\
\hline
\end{tabular}

a Negative values are in emission.

and February 2019. A summary of these observations is given in Table 1 . The B3000 and R7000 gratings provided wavelength coverage of $3500-6000 \AA(\lambda / \Delta \lambda \sim 3000)$ and 5300 $7000 \AA(\lambda / \Delta \lambda \sim 7000)$, respectively. The raw data were reduced using the PYTHON-based data reduction pipeline, PyWiFeS (Childress et al. 2014). The reduction process includes bad pixel repair, bias, and dark current subtraction, flat fielding, wavelength calibration, flux calibration, and data cube creation. The wavelength calibration was performed using a series of Ne-Ar arc lamp exposures, taken throughout the night.

We compared the R7000 spectrum against the M-type spectral templates of Bochanski et al. (2007) that were generated by utilizing more than 4,000 M-dwarfs spectra from the Sloan Digital Sky Survey (SDSS; York et al. 2000). Visual comparison of the 2M1546 spectra against a set of Mdwarf template spectra shows the best match at M5. 2M1546 is located well outside of any molecular cloud and, at a distance of only $60 \mathrm{pc}$, it should have negligible interstellar extinction. Nevertheless, the accretion causes "veiling", in which the accretion shock increases flux in the UV/optical, while the surrounding accretion disc produces additional flux to the photosphere in the IR (Vacca \& Sandell 2011). Veiling can potentially affect our spectral type estimation because it will change the spectral shape (Herczeg \& Hillenbrand 2008; Manara et al. 2013; Ingleby et al. 2014). However, a change in the spectral shape due to veiling occurs usually over a wide wavelength range while our spectral type estimate is focused on rather narrow wavelength ranges of 100 to $200 \AA$ (e.g., shaded regions of 1 ). Therefore, our estimate of M5 spectral type should not be far from the truth and appropriate for the discussion in this paper. A more robust, unambiguous spectral type estimate can be done in the future by using a high spectral resolution echelle spectrum by measuring line ratios of some temperature sensitive lines that are adjacent in wavelengths so that veiling effect can be negligible.

The Li $6708 \AA$ absorption feature was strongly detected in both epochs of WiFES spectra (EW of 430 and $600 \mathrm{~m} \AA$ ). Considering a typical equivalent width measurement uncertainty $(\sim 50 \mathrm{~mA})$ in our WiFES spectra, the observed difference is likely a real variability of the $\mathrm{Li} 6708$ line strength and related to a variable veiling. For an M5 star, the detected Li 6708 line strength indicates the undepleted level of Lithium in the atmosphere while a "normal" $55 \mathrm{Myr}$ old M5 star should have depleted all Lithium already. An ongoing accretion must have replenished Lithium in the atmosphere so that the 6708 line was strongly detected in our observation.

\section{$2.1 \quad$ Emission Lines}

The magnetospheric accretion of the disc occurs from the inner edge of the disc onto the surface of the central source. This supersonic flow has nearly free-fall velocities, resulting in large line widths of some emission lines. In the region of the accretion shock, on the other hand, some narrow emission lines are more likely produced (Hartmann et al. 2016). The strength and/or shape of some lines such as hydrogen recombination lines and $\mathrm{He}$ I, Ca II, and Na I lines are known as accretion tracers (see e.g., Muzerolle et al. 1998; Antoniucci et al. 2011; Biazzo et al. 2014). The observed spectra of $2 \mathrm{M} 1546$ cover $\mathrm{H} \alpha$ and $\mathrm{He}$ I lines.

Both WiFeS observations show strong $\mathrm{H} \alpha$ emission, with equivalent widths in excess of $100 \AA$. However, because of the contrast effect caused by the depression of the stellar continuum by molecular absorption, the equivalent widths of $\mathrm{H} \alpha$ lines $(\mathrm{EW}(\mathrm{H} \alpha))$ in non-accreting stars are typically enhanced at later spectral types (White \& Basri 2003; Martín et al. 1998; Basri \& Marcy 1995). Barrado y Navascués \& Martín (2003) presented an $\mathrm{EW}(\mathrm{H} \alpha)$ accretion criterion as a function of spectral type, where the upper limit for accretors is $\mathrm{EW}(\mathrm{H} \alpha) \sim-20 \AA$ at M5. This was corroborated by Duchêne et al. (2017) after an extensive analysis of known T Tauri stars. With equivalent widths of $-210 \AA$ and $-120 \AA$, 2M1546 clearly exceeds this criterion.

Although the contrast effect is considered, the evaluation of accretion based on $\mathrm{H} \alpha$ strength has a caveat. The chromospheric activity-typically enhanced for young starsgenerates a strong $\mathrm{H} \alpha$ emission similar to the case of accretion. The line profile of $\mathrm{H} \alpha$ is used for distinguishing between accreting and nonaccreting objects: disc accretion generates broad and asymmetric $\mathrm{H} \alpha$ lines (Luhman et al. 2007). Fig. 2 shows the $\mathrm{H} \alpha$ velocity profiles for $2 \mathrm{M} 1546$, which are asymmetric in both two observations. The asymmetric feature can be explained by the inclination effects and/or absorption by an accompanying outflowing wind (Mohanty et al. 2005; Alencar \& Basri 2000). A quantitative diagnostic value using $\mathrm{H} \alpha$ emission line profile is the full width of $\mathrm{H} \alpha$ at 10 per cent of the line peak $\left(v_{10}\right)$ (White \& Basri 2003; Mohanty et al. 2005). However, the broadened $\mathrm{H} \alpha$ line profile is not always explained by the accretion. Fast rotator and binarity can broaden the $\mathrm{H} \alpha$ emission line, which can be misdiagnosed as an accretor (Manara et al. 2013). In the opposite way, the inclination of the accretion disc can produce a narrower emission line along the line of sight, which can be misdiagnosed as a nonaccretor (Mohanty et al. 2005). Published $v_{10}$ accretion criteria vary from $200<v_{10}<270$ $\mathrm{km} \mathrm{s}^{-1}$ (Fang et al. 2013; Jayawardhana et al. 2003; White $\&$ Basri 2003), independent of spectral type. Measured $v_{10}$ values for $2 \mathrm{M} 1546$ are over $300 \mathrm{~km} \mathrm{~s}^{-1}$ (Table 1), which exceeds the strictest accretion criterion (i.e., $>270 \mathrm{~km} \mathrm{~s}^{-1}$ ). Using the $v_{10}$ value and the accretion rate $\left(\dot{M}_{\text {acc }}\right)$ relation 


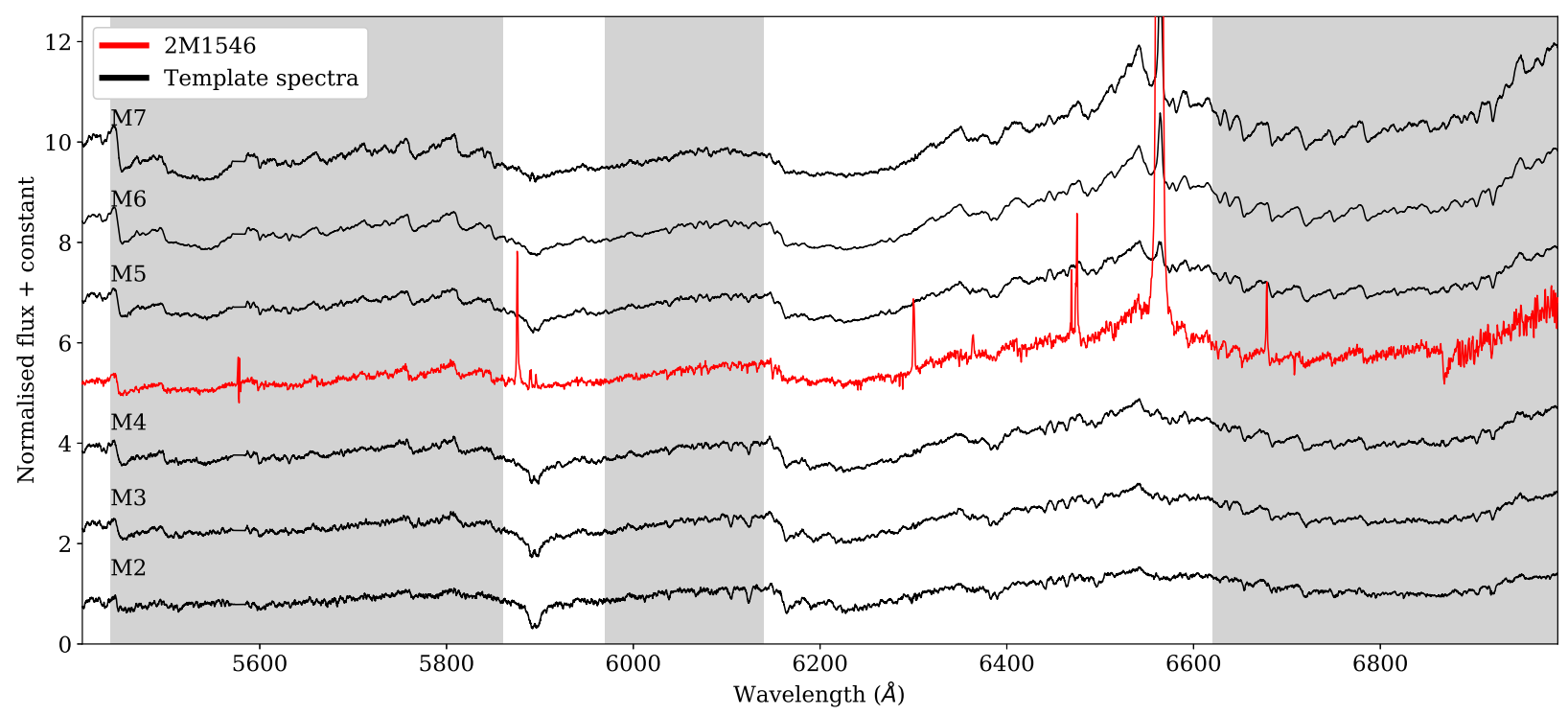

Figure 1. R7000 spectrum of 2M1546 (red) compared to the M-type templates from Bochanski et al. (2007). The templates are mean spectra of four thousand dwarfs from the Sloan Digital Sky Survey (SDSS; York et al. 2000). For details of creating these template spectra, see Bochanski et al. 2007. The spectrum of $2 \mathrm{M} 1546$ and the templates were normalised at $5800 \AA$ prior to plotting. In the spectral type determination, the overall shape of the target and templates are compared focused more on the gray shaded area.

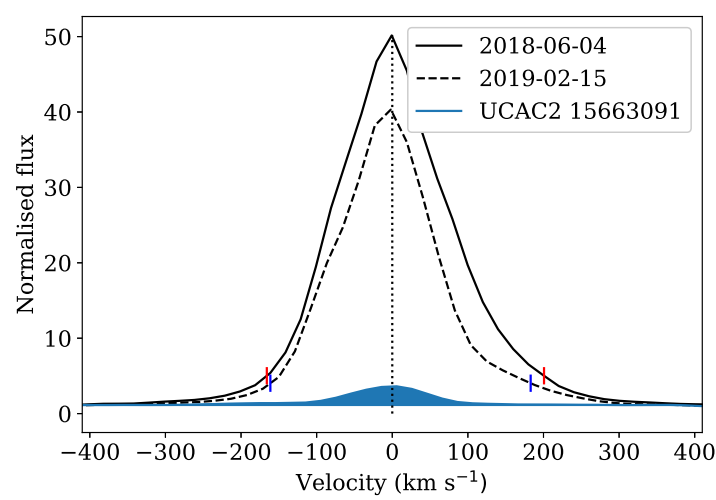

Figure 2. WiFeS/R7000 $\mathrm{H} \alpha$ velocity profiles of $2 \mathrm{M} 1546$. Small vertical lines (red and blue) indicate 10 per cent of the peak flux used for measuring the $v_{10}$ value. The WiFeS line profile of the M5 non-accretor UCAC2 15663091 is also plotted for comparison. All spectra are shifted to the heliocentric rest frame.

of Natta et al. (2004), we estimate $\dot{M}_{\text {acc }}=1.3 \times 10^{-10} \mathrm{M}_{\odot}$ $\mathrm{yr}^{-1}$. Considering many M-type pre-MS stars found to have accretion rates as low as $\sim 2 \times 10^{-12} \mathrm{M}_{\odot} \mathrm{yr}^{-1}$ (Alcalá et al. 2014; Herczeg et al. 2009), the rate we infer for $2 \mathrm{M} 1546$ suggests it is actively accreting from its inner disc.

From our SED fitting, we obtain the bestfit stellar radius $\left(0.461 R_{\odot}\right)$, effective temperature $(2940 \mathrm{~K})$, and luminosity $\left(0.014 L_{\odot}\right)$. We alert readers that this SED fitting ignores any effects from veiling and self-extinction so that they should be taken with caution. Based on Baraffe et al. (2015), these values are inconsistent with values from a $\sim 55 \mathrm{Myr}$ old M5 star. These bestfit SED parameters are instead well matched for parameters of a $10 \mathrm{Myr}$ old mid-M type star with mass of $0.1 M_{\odot}$. For this mass, a typical mass accre- tion rate is expected to be $1.0 \times 10^{-10} \mathrm{M}_{\odot} \mathrm{yr}^{-1}$ (Hartmann et al. 2016) which agrees well with our obtained accretion rate. As shown in the following section, 2M1546 is a highly likely member of the $55 \mathrm{Myr}$ old Argus association. When a star has prolonged accretion, then it may be conceivable that such a star has stellar parameters of a younger star because of the accretion effect on its evolution. One needs higher spectal resolution data to confirm this possible effect of accretion by independently obtaining a spectral type not affected by veiling.

Emission in He I $\lambda 6678$ is also reasonably well correlated with accretion among very low mass stars and brown dwarfs (Mohanty et al. 2005; Alcalá et al. 2014, 2017). Gizis et al. (2002) showed that He I $\lambda 6678$ is generally present in low levels $(|\mathrm{EW}(\mathrm{He} \mathrm{I})| \ll 1 \AA)$ in older chromosphericallyactive stars. 2 M1546 shows strong He I $\lambda 6678$ emission at both epochs (EWs of $-0.8 \AA$ and $-1.6 \AA$ ), supporting the hypothesis of ongoing accretion (See Table 1 and Fig. 4). In addition to strong $\mathrm{H} \alpha$, we also detect other Balmer line emission up to H17 (Fig. 3).

As shown in Figs. 1 and 4, we detect strong [O I] emission at $6300 \AA$ at both epochs. Studies investigating the prominent [O I] $\lambda 6300$ emission toward young stellar objects suggest that jets, MHD winds, photoevaporative winds, and photodissociation of $\mathrm{OH}$ in the disc surface layers are the possible origins of the line (e.g, Banzatti et al. 2019; Natta et al. 2014; Rigliaco et al. 2013; Acke et al. 2005; Störzer \& Hollenbach 2000). Störzer \& Hollenbach (2000) predicts that [O I] $\lambda 6300$ is dominated by $\mathrm{OH}$ photodissociation in the region with a line ratio [O I] $\lambda 6300 /[\mathrm{O}$ I] $\lambda 5577$ smaller than 10 . Our spectra show a potentially weak $(\sim 1 \AA)$ detection of [O I] $\lambda 5577$ which results in the line ratio of about 3 . This small line ratio favours the other explanations for the origin of OI emission such as from winds and/or jets. 


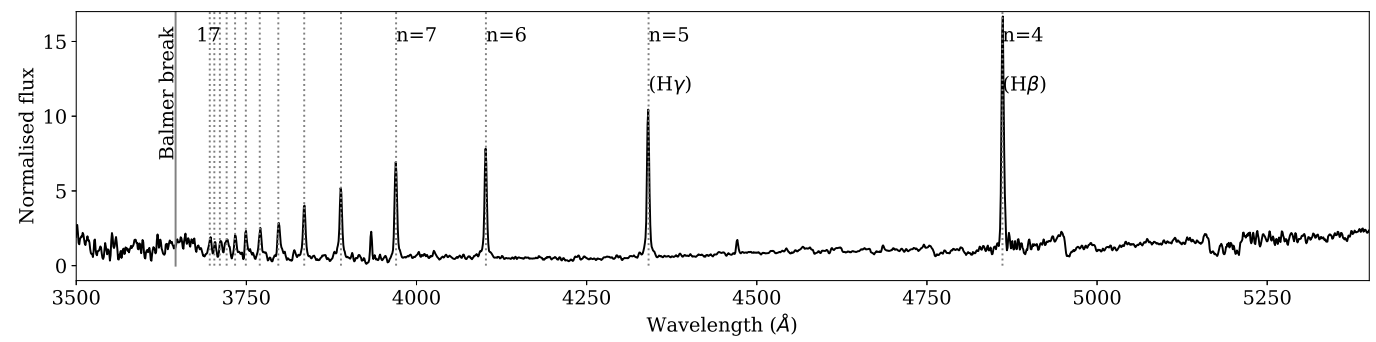

Figure 3. The B3000 spectrum of 2MJ1546 showing Balmer series in emission up to $n=17$.

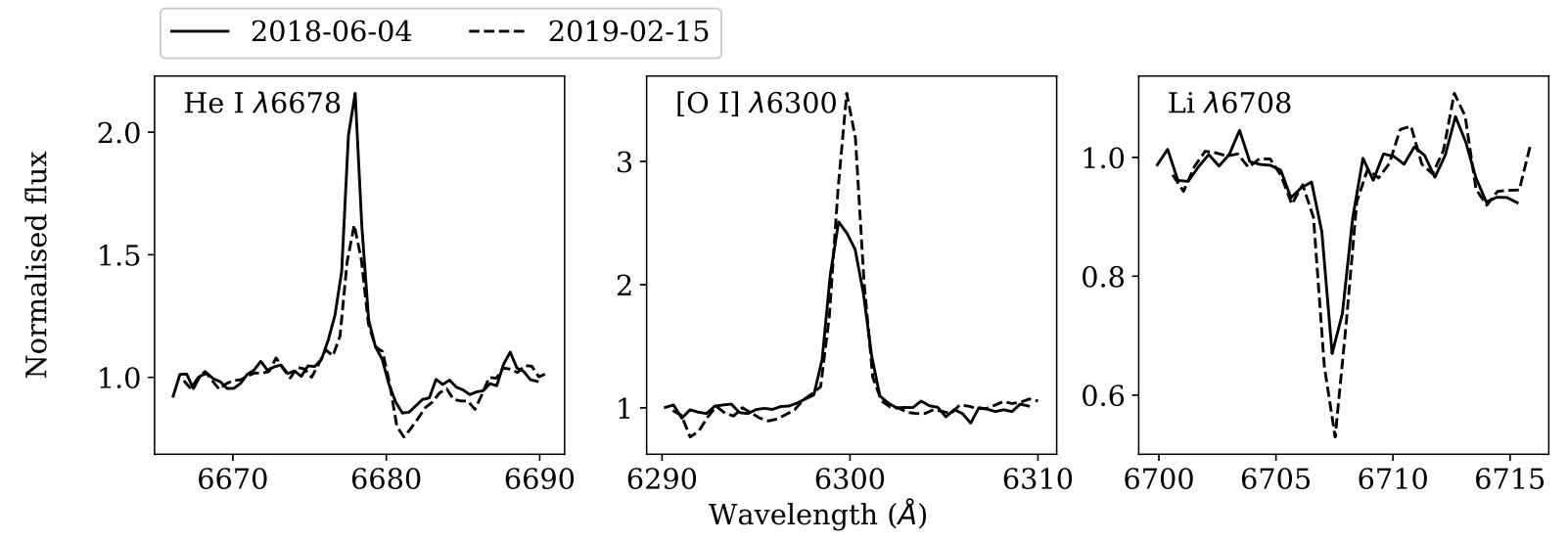

Figure 4. WiFeS/R7000 line profiles of He I $\lambda 6678$, [O I] $\lambda 6300$, and Li I $\lambda 6708$ at the two observation epochs.

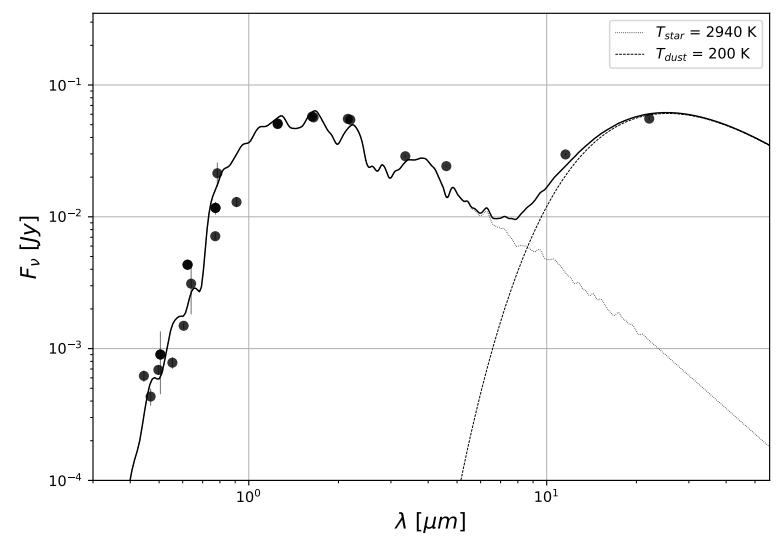

Figure 5. Spectral energy distribution for $2 \mathrm{M} 1546$ with the bestfitting photosphere model of $\boldsymbol{T}_{\mathrm{star}}=2940 \mathrm{~K}$ (dotted line) and a 200 $\mathrm{K}$ blackbody (dashed line) combined to produce a total model (solid line).

\section{CIRCUMSTELLAR DISC EMISSION}

Dust emission from the circumstellar disc is observed as the IR excess of the spectral energy distribution (SED). The SED of 2M1546 was generated utilizing catalogue data from POSS-II ( $F F N$; Cabanela et al. 2003), SkyMapper DR1 (griz; Wolf et al. 2018), Gaia DR2 ( $\left.G_{\mathrm{BP}}, G_{\mathrm{RP}}, G\right)$, 2MASS $\left(\mathrm{JHK}_{S}\right.$; Cutri et al. 2003), and All WISE (W1-W4; Cutri et al. 2014). Examinations of high angular resolu- tion optical/near-IR images show no possible contaminating source within the spatial resolution of WISE images. Therefore, we conclude that there is no contamination and these photometric data are only from 2M1546. We fit synthetic photometry derived from BT-Settl (Allard et al. 2012) using our SED fitting technique described in Rhee et al. (2007). The resulting SED and the best-fitting model are presented in Fig. 5.

The best-fitting temperature $\left(T_{\text {star }}=2940 \mathrm{~K}\right)$ is consistent with the pre-MS temperature scale for young (1030 Myr) stars derived by Pecaut \& Mamajek (2013) for a spectral type of M5. We can satisfactorily fit the WISE W3 $(12 \mu \mathrm{m})$ and $W 4(22 \mu \mathrm{m})$ excesses with a single blackbody of temperature $T_{\text {dust }} \sim 200 \mathrm{~K}$. With little excess emission at $\lambda<5 \mu \mathrm{m}$ and a significant excess at $\lambda>10 \mu \mathrm{m}$ we classify 2M1546 as having a transitional disc (Strom et al. 1989; Williams \& Cieza 2011), with clearly some material in the inner disc remaining to drive the accretion we observed.

Murphy et al. (2018) investigated the disc surrounding the M5 Carina member WISE J080822.18-644357.3 (hereafter WISE J0808), which has a similar SED and accretion characteristics to $2 \mathrm{M} 1546$. They found a cold disc component which was well fitted by a $\sim 240 \mathrm{~K}$ blackbody resulting from populations of small dust grains released by sublimating planetesimals. Flaherty et al. (2019) recently observed WISE J0808 with the Atacama Large Millimetre/sub-millimetre Array (ALMA) but did not detect any CO gas, indicating WISE J0808 may be at an evolutionary state between a gas-rich transition disc and a gas-poor second-generation debris disc. Considering the 
similarities between WISE J0808 and 2M1546, the latter is also probably a borderline transition/debris disc accreting the last of its inner gas on to the star. More observations, including from ALMA, are required before definitively assessing the evolutionary stage of the disc around 2M1546.

\section{AGE ESTIMATION BASED ON MOVING GROUP MEMBERSHIP PROBABILITY}

One of the key parameters for constraining disc models is stellar age and, for young nearby stars within $150 \mathrm{pc}$, one of the most reliable age-dating methods is to test whether the star is a member of one of several young moving groups in the solar neighborhood with well determined ages (e.g. Zuckerman \& Song 2004; Torres et al. 2008). Although the absolute age scales from these moving groups should not be overinterpreted, relative age ordering of several major moving groups is secure and can be used to derive ages of member stars at mugh higher precision than possible for an isolated star. The relative age rank for major moving groups are: TWA $<$ BPMG $<$ Columba/Carina $<$ Argus $<$ AB Dor (Zuckerman \& Song 2004; Torres et al. 2008; Gagné et al. 2014; Bell et al. 2015). We note that the existence of the Argus group was challenged by Bell et al. (2015) and Riedel et al. (2017), but Zuckerman (2019) reaffirm the Argus moving group.

There are several moving group membership probability calculation tools available in the literature (e.g. BANYAN II $\& \Sigma$ and BAMG 1 \& 2; Gagné et al. 2014, 2018; Lee \& Song 2018, 2019). The main differences between these tools are their internal membership lists and how they calculate the structural properties (mean $X Y Z$ positions, $U V W$ velocities and their distributions) of each moving group. BAMG 2 is the most recent code for calculating kinematic memberships and was developed to be self-consistent with respect to group memberships (Lee \& Song 2018). For this reason we adopt membership probabilities from it over those from other tools.

Table 2 presents membership probabilities using our two measured RV values. BAMG 2 evaluates 2M1546 as an Argus member while the two BANYAN models provide a very small Argus membership probability, with the star most likely being a field object. If 2M1546 was an old field star, then its ongoing accretion is even more interesting yet more challenging to understand. If an isolated accreting preMS star, then 2M1546 belongs to a rare group of nearby ( $\lesssim 60 \mathrm{pc}$ ) youngest stars such as some accreting TWA members. Fig. 6 compares the heliocentric position $(X Y Z)$ and velocity $(U V W)$ of $2 \mathrm{M} 1546$ to the moving group models used by BAMG 2 . The position and velocity of $2 \mathrm{M} 1546$ are well matched to the $\sim 55$ Myr-old Argus group, as expected from the membership probabilities in Table 2 .

\section{DISCUSSION AND CONCLUSIONS}

We summarize the important properties of 2M1546 from the literature and this work in Table 3 . The proximity $(59 \mathrm{pc})$, late spectral type (M5), old age (55 Myr), mid-IR excess emission, and ample evidence of on-going accretion observed in 2M1546 make it an interesting laboratory for studying prolonged disc accretion. It is the oldest known accreting star to date, and can therefore be used for studying the upper limit on the lifetimes of gas-rich discs. The kinematic properties of the star are consistent with the Argus moving group and its estimated age of $\sim 55 \mathrm{Myr}$.

Over the past several years, several mid-M type accretors at various ages have been reported in the literature (Table 4). All of the stars in Table 4 show strong mid-IR excess emission at $W 3$ and $W 4$ and signs of ongoing accretion. Among these sources, WISE J0808 appears to be the most similar to $2 \mathrm{M} 1546$, with $v_{10}$ velocity widths in excess of $300 \mathrm{~km} \mathrm{~s}^{-1}$. 2MASS J1239-5702 and 2MASS J1422-3623 have slightly weaker $\mathrm{H} \alpha$ equivalent widths and $v_{10}$ values comparble to 2M1546. However, they are believed to be younger (10-17 Myr) members of LCC or UCL subgroups of the Sco-Cen OB association (Murphy et al. 2015). While LDS $5606 \mathrm{~A}+\mathrm{B}$ are also actively accreting sources, they are binaries and hence their accretion might have been affected by their companions. 2MASS J1337-4736 is claimed to be accreting based on its asymmetric $\mathrm{H} \alpha$ profile and WISE excess (Rodriguez et al. 2011; Schneider et al. 2012b). Zuckerman (2015) found that this star has a distant companion. While not having a measured $v_{10}$ value, TWA 31 has a strong $\mathrm{EW}(\mathrm{H} \alpha)$ value and significant excess at $W 3$ and $W 4$, supporting an accretion hypothesis (Schneider et al. 2012a; Zuckerman 2015). The remaining three stars seem to show marginal signs of accretion. 2MASS J0844-7833 and 2MASS J0508-2101 barely exceed the lower limit for accretion criterion in terms of $v_{10}$ and/or $\mathrm{EW}(\mathrm{H} \alpha)$. Compared to other accretion sources, 2MASS J0501-4337 also has a significantly lower $\operatorname{EW}(\mathrm{H} \alpha)$ and a $v_{10}$ width that barely exceeds the accretion criterion of Barrado y Navascués \& Martín (2003).

With the exception of WISE J0808, the inferred ages for these stars are all smaller than the age of the $\beta$ Pic Moving Group $(\lesssim 25 \mathrm{Myr})$. Therefore, the slightly prolonged accretion at these stars could have been accepted as an unusual phenomenon seen in some rare outliers. Now, we see at least a handful of adolescent mid-M type stars with clear signs of ongoing accretion, suggesting that accretion can be maintained for several tens of million years around low-mass stars under certain conditions. The discovery of these older M-type accretors could eventually reveal a less efficient process of removing circumstellar gas and dust around low-mass stars. We note that the plethora of M5 accretors at ages of 30-55 Myr coincides with the mass boundary at which stars become fully convective in their interiors.

\section{ACKNOWLEDGEMENTS}

We thank Michael Bessell for obtaining the second epoch B3000 and R7000 spectra of 2M1546 in 2019 February. This work was supported by the Basic Science Research Program through the National Research Foundation of Korea (grant No. NRF-2018R1A2B6003423).

\section{REFERENCES}

Acke B., van den Ancker M. E., Dullemond C. P., 2005, A\&A, 436,209

Alcalá J. M. et al., 2014, A\&A, 561, 2

Alcalá J. M. et al., 2017, A\&A, 600, 20

Alencar S., Basri G., 2000, AJ, 119, 1881 
Table 2. Kinematic membership probabilities.

\begin{tabular}{lll}
\hline & & Membership probability: Group (per cent) \\
\cline { 2 - 3 } RV $\left(\mathrm{km} \mathrm{s}^{-1}\right)$ & $-4.4 \pm 0.9$ & $-3.5 \pm 1$ \\
BAMG 2 & $\operatorname{Argus}(95)+\operatorname{Field}(5)$ & $\operatorname{Argus}(83)+\operatorname{BPMG}(14)$ \\
BANYAN II & Field(78) $+\operatorname{Argus}(22)$ & Field $(89)+\operatorname{Argus}(9)$ \\
BANYAN $\Sigma$ & Field(94) $+\operatorname{Argus}(2)$ & Field $(87)+$ UCL $(8)+\operatorname{Argus}(5)$ \\
\hline
\end{tabular}

Note. Since only groups with membership probabilities $>2$ per cent are presented here, the sum of probabilities is smaller than 100 per cent.

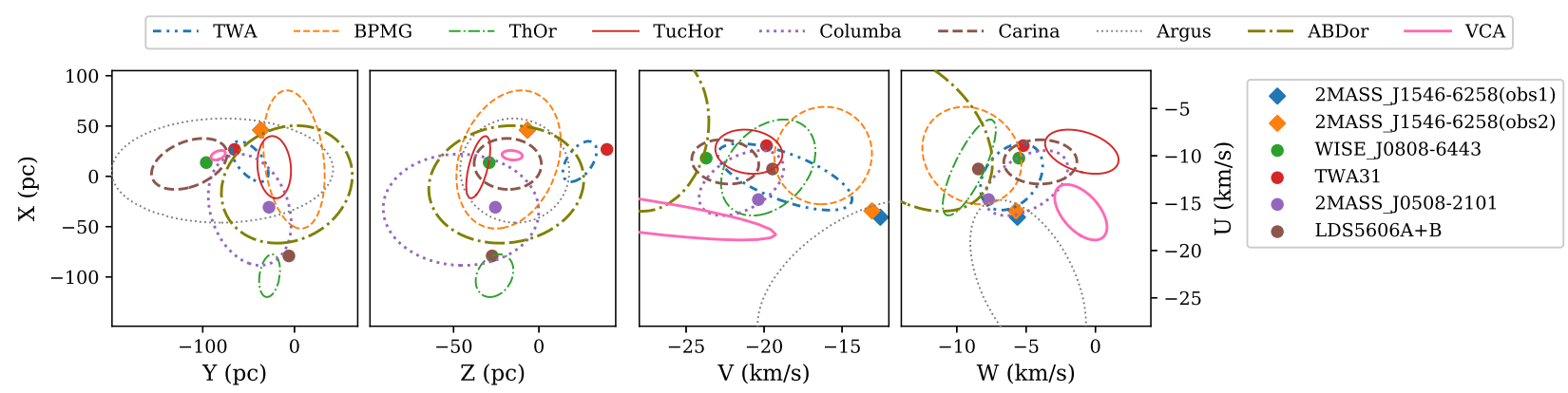

Figure 6. Accreting mid-M type stars and models of NYMGs in the XYZUVW spaces.

Table 3. Summary of 2M1546 parameters.

\begin{tabular}{lcc}
\hline Parameter & Value & Units \\
\hline R. A. & $15: 46: 07.52^{\mathrm{a}}$ & hh:mm:ss.ss \\
Dec. & $-62: 58: 04.2^{\mathrm{a}}$ & dd:mm:ss.s \\
SpT & $\mathrm{M} 5$ & \\
$\mu_{\alpha} \cos \delta$ & $-42.7 \pm 0.1^{\mathrm{a}}$ & $\mathrm{mas} \mathrm{yr}^{-1}$ \\
$\mu_{\delta}$ & $-61.5 \pm 0.1^{\mathrm{a}}$ & $\mathrm{mas} \mathrm{yr}^{-1}$ \\
Distance & $59.2 \pm 0.3^{\mathrm{a}}$ & $\mathrm{pc}^{-1}$ \\
RV & $-4.4,-3.5$ & $\mathrm{~km} \mathrm{~s}^{-1}$ \\
$T_{\text {eff }}$ & 2940 & $\mathrm{~K}$ \\
$\log \left(L / L_{\odot}\right)$ & -1.83 & $\mathrm{dex}^{-1}$ \\
$\dot{M}_{\text {acc }}$ & $1.31^{-10}$ & $\mathrm{M}_{\odot} \mathrm{yr}^{-1}$ \\
Mass & 0.11 & $\mathrm{M}_{\odot}$ \\
Age & 55 & $\mathrm{Myr}$ \\
$X, Y, Z$ & $45.9,-36.7,-6.7$ & $\mathrm{pc}^{-1}$ \\
$U, V, W$ & $-16.5,-12.5,-5.6^{\mathrm{b}}$ & $\mathrm{km} \mathrm{s}^{-1}$ \\
& $-15.8,-13.1,-5.7^{\mathrm{c}}$ & $\mathrm{km} \mathrm{s}^{-1}$ \\
\hline
\end{tabular}

a Gaia Collaboration et al. (2018)

b Using RV $=-4.4 \mathrm{~km} \mathrm{~s}^{-1}$

${ }^{\mathrm{c}}$ Using RV $=-3.5 \mathrm{~km} \mathrm{~s}^{-1}$

Allard F., Homeier D., Freytag B., 2012, Phil. Trans. R. Soc. A., 370,2765

Antoniucci S. et al., 2011, A\&A, 534, 32

Banzatti A., Pascucci I., Edwards S., Fang M., Gorti U., Flock M., 2019, ApJ, 870, 76

Baraffe I., Homeier D., Allard F., Chabrier G., 2015, A\&A, 577, 42

Barrado y Navascués D. \& Martín E. L., 2003, AJ, 126, 2997

Basri G., Marcy G. W., 1995, AJ, 109, 762

Bell C. P. M., Mamajek E. E., Naylor T., 2015, MNRAS, 454, 593

Biazzo K., Alcalá J. M., Frasca A., Zusi M., Getman F., Covino E., Gandolfi D., 2014, A\&A, 572, 84

Bochanski J., West A., Hawley L., Covey R., 2007, AJ, 133, 531

Boucher A., Lafrenière D., Gagné J., Malo L., 2016, ApJ, 832, 50

Cabanela J. E., Humphreys R. M., Aldering G., Larsen J. A., Odewahn S. C., Thurmes P. M., Cornuelle C. S., 2003, PASP,
115,837

Childress M. J., Vogt F. P. A., Nielsen J., Sharp R. G., 2014, Ap\&SS, 349, 617

Cutri R. M., Skrutskie M. R., van Dyk S. et al., 2003, 2MASS All Sky Catalog of point sources. Available at http://www.ipac.caltech.edu/2mass/

Cutri R. M., et al., 2014, VizieR Online Data Catalog, 2328

Dopita M., Hart J., McGregor P., Oates P., Bloxham G., Jones D., 2007, Ap\&SS, 310, 255

Duchêne G, Becker A., Yang Y., Bouy H., De Rosa R., Patience J., Girard J. H., 2017, MNRAS, 469, 1783

Esplin T. L., Luhman K. L., Mamajek E. E., 2014, ApJ, 784, 126

Fang M., Kim J. S., van Boekel R., Sicilia-Aguilar A., Henning T., Flaherty K., 2013, ApJS, 207, 5

Flaherty K., Hughes A. M., Mamajek E. E., Murphy S. J., 2019, ApJ, 872, 92

Gagné J., Lafrenière D., Doyon R., Malo L., Artigau É, 2014, ApJ, 783, 121

Gagné J. et al., 2018, ApJ, 856, 23

Gaia Collaboration et al., 2018, A\&A, 616, 1

Gizis J. E., Reid I. N., Hawley S. L., 2002, AJ, 123, 3356

Greaves J. S., Rice W. K. M., 2010, MNRAS, 407, 1981

Haisch Jr. K. E., Lada E. A., Lada C. J., 2001, ApJ, 553, 153

Hartmann L., Herczeg G., Calvet N., 2016, ARA\&A, 54, 135

Herczeg G. and Hillenbrand L. A.,2008, ApJ, 681, 594

Herczeg G., Cruz K. L., Hillenbrand L. A., 2009, ApJ, 696, 1589

Ingleby L. et al., 2014, ApJ, 790, 47

Jayawardhana R., Mohanty S., Basri G., 2003, ApJ, 592, 282

Lee J., Song I., 2018, MNRAS, 475, 2955

Lee J., Song I., 2019, MNRAS, 486, 3434

Luhman K. L., Mamajek E. E., 2012, ApJ, 758, 31

Luhman K. L., Joergens V., Lada C., Muzerolle J., Pascucci I., White R., 2007, Protostars and Planets V., University of Arizona Press, Tucson, p. 443

Mamajek E. E., Meyer M. R., Liebert J., 2002, AJ, 124, 1670

Mamajek E. E., 2009, in Usuda T., Tamura M., Ishii M., eds, API Conf. Proc. Vol. 1158, Exoplanets And Disks: Their Formation And Diversity. Am. Inst. Phys., New York, p. 3

Manara C. F. et al., 2013, A\&A, 551, 107

Manara C. F., Morbidelli A., Guillot T., A\&A, 618, 3

Martín E., Montmerle T., Gregorio-Hetem J., Casanova S., 1998, 
Table 4. Accreting mid-M type stars from the literature.

\begin{tabular}{|c|c|c|c|c|c|c|c|c|}
\hline Name & $\mathrm{SpT}$ & $\begin{array}{l}\text { Dist. } \\
\text { (pc) }\end{array}$ & $\begin{array}{c}\mathrm{RV} \\
\left(\mathrm{km} \mathrm{s}^{-1}\right)\end{array}$ & $\begin{array}{c}\mathrm{EW}(\mathrm{H} \alpha) \\
(\AA)\end{array}$ & $\begin{array}{c}v_{10}[\mathrm{H} \alpha] \\
\left(\mathrm{km} \mathrm{s}^{-1}\right)\end{array}$ & $\begin{array}{l}\text { Age from Lit. } \\
\text { (Myr; group) }\end{array}$ & $\begin{array}{l}\text { Age from this work }{ }^{\mathrm{a}} \\
\text { (Myr; group) }\end{array}$ & Refs \\
\hline 2MASS J08440915-7833457 & M4.5 & 98 & - & 60 & 212 & 8-14 ( $\eta$ Cha) & $-\mathrm{b}$ & 1 \\
\hline TWA 31 & $\mathrm{M} 4.2$ & 81 & $10.5 \pm 0.4$ & 115 & - & 7-13 (TWA) & 7-13 (TWA) & 2 \\
\hline 2MASS J13373839-4736297 & M3.5 & 126 & - & 13.7 & - & $10-17(\mathrm{LCC})$ & $-\mathrm{b}$ & 3,4 \\
\hline 2MASS J12392312-5702400 & M5 & 180 & $16 \pm 2$ & $27-63$ & $238-331$ & $10-17$ (LCC) & $-\mathrm{b}$ & 5 \\
\hline 2MASS J14224891-3623009 & M5 & - & $9 \pm 2$ & $33-91$ & $236-341$ & $10-16$ (UCL) & $-\mathrm{b}$ & 5 \\
\hline 2MASS J05082729-2101444 & M5 & 49 & $24.9 \pm 0.9$ & 25 & 197 & $12-25$ (BPMG) & 30-44 (Columba) & 1 \\
\hline LDS $5606 \mathrm{~A}$ & M5 & 84 & $14.9 \pm 0.8$ & $99-135$ & 250 & $12-25$ (BPMG) & 30-44 (Columba) & 6,7 \\
\hline LDS $5606 \mathrm{~B}$ & M5 & 84 & $14.9 \pm 0.4$ & 25 & 135 & $12-25$ (BPMG) & 30-44 (Columba) & 6,7 \\
\hline 2MASS J05010082-4337102 & M4.5 & - & _- & 7.6 & 210 & 30-44 (Columba) & ${ }^{\mathrm{c}} \mathrm{c}$ & 8 \\
\hline WISE J080822.18-644357.3 & M5 & $90^{\mathrm{d}}$ & $22.7 \pm 0.5$ & $65-125$ & $300-350$ & 30-49 (Carina) & 30-49 (Carina) & 9 \\
\hline 2MASS J15460752-6258042 & M5 & 59 & $-4.4,-3.5$ & $120-210$ & $310-366$ & - & 40-69 (Argus) & 10 \\
\hline J0446A & M6 & 83 & $26.7 \pm 16.8$ & 10.4 & 210 & 30-44 (Columba) & - & 11 \\
\hline J0446B & M6 & 82 & $29.8 \pm 16.8$ & 16.8 & 239 & 30-44 (Columba) & - & 11 \\
\hline J0949A & M4 & 79 & $22.4 \pm 16.7$ & 110 & 367 & 30-49 (Carina) & - & 11 \\
\hline J0949B & M5 & 78 & $20.5 \pm 16.8$ & 24 & 305 & 30-49 (Carina) & - & 11 \\
\hline
\end{tabular}

a Ages are obtained based on the moving group membership probabilities calculated using BAMG 2 except where marked.

${ }^{\mathrm{b}} \eta$ Cha, LCC, and UCL are not included in BAMG 2. BANYAN $\Sigma$ suggests that 2MASS J0844-7833, J1337-4736, J1239-5702, and J14223623 are likely members of $\eta$ Cha $(P=62$ per cent $)$ LCC $(P=63$ per cent $)$ LCC $(P=52$ per cent $)$, and UCL $(P=84$ per cent $)$, respectively.

c Since a Gaia proper motion for this star not exist, the Bayesian membership probability cannot be calculated.

d Kinematic distance based on membership in Carina (Murphy et al. 2018).

Notes. References for group age: $\eta$ Cha- Bell et al. 2015; TWA-Bell et al. 2015; LCC- Song et al. 2012, Pecaut \& Mamajek 2016; UCL- Song et al. 2012, Pecaut \& Mamajek 2016; BPMG- Song \& Zuckerman 2003, Bell et al. 2015; Columba- Torres et al. 2008, Bell et al. 2015; Carina- Torres et al. 2008, Bell et al. 2015; Argus- Bell et al. 2015, Zuckerman 2019

References. (1) Schneider et al. 2019; (2) Schneider et al. 2012a; (3) Schneider et al. 2012b; (4) Rodriguez et al. 2011; (5) Murphy et al. 2015; (6) Rodriguez et al. 2014; (7) Zuckerman et al. 2014; (8) Boucher et al. 2016; (9) Murphy et al. 2018; (10) This work.; (11) Silverberg et al. 2020

MNRAS, 300, 733

Najita J. R., Kenyon S. J., 2014, MNRAS, 445, 3315

Natta A., Testi L., Alcalá J. M., Rigliaco E., Covino E., Stelzer B., D'Elia V., 2014, A\&A, 569, 5

Miszalski, B., \& Mikołajewska, J. 2014, MNRAS, 440, 1410

Mohanty U., Jayawardhana R., Basri G., 2005, ApJ, 626, 498

Moór A. et al. 2011, ApJ, 740, 7

Murphy S. J., Lawson W. A., Bento J., 2015, MNRAS, 453, 2220

Murphy S. J., Mamajek E. E., Bell C. P. M., 2018, MNRAS, 476, 3290

Muzerolle J., Hartmann L., Calvet N., 1998, AJ, 116, 2965

Muzerolle J., Brinceño C., Calvet N., Hatmann L., Hillenbrand L., Gullbring E., 2000 ,ApJ, 545, 141

Natta A., Testi L., Muzerolle J., Randish S., Comerón F., Persi P., 2004, A\&A, 424, 603

Pecaut M. J., Mamajek E. E., 2013, ApJS, 208, 9

Pecaut M. J., Mamajek E. E., 2016, MNRAS, 461, 794

Pfalzner S., Bannister M. T., 2019, ApJ, 874, 34

Rhee J. H., Song I., Zuckerman, B., McElwain, M., 2007, ApJ, 660,1556

Ribas Á., Bouy H., Merín B., 2015, A\&A, 576, 52

Riedel A. et al., 2017, AJ, 153, 95

Rigliaco E., Pascucci I., Gorti U., Edwards S., Hollenbach D., ApJ, 772, 60

Rodriguez D. R., Zuckerman B., Kastner J. H., Bessell M. S., Faherty J. K., Murphy S. J., 2013, ApJ, 774, 101

Rodriguez D. R., Zuckerman B., Faherty J. K., Vican L., 2014, A\&A, 567, 20

Schneider A. C., Melis C., Song I., 2012a, ApJ, 754, 39

Schneider A. C., Song I., Melis C., Zuckerman B., Bessell M., 2012b, ApJ, 757, 163

Schneider A. C., Shkolnik E. L., Allers K. N., Kraus A. L., Liu M. C., Weinberger A. J., Flagg L., 2019, AJ, 157, 234

Silverberg S. M. et al., 2016, ApJ, 830, 28
Silverberg S. M. et al., 2020, ApJ, 890, 106

Song I., Zuckerman B., 2003, ApJ, 599, 342

Song I., Zuckerman B., Bessell M. S., 2012, AJ, 144, 8

Stoörzer H., Hollenbach D., ApJ, 2000, 539, 751

Strom K. M., Strom S. E., Edwards S., Cabrit S., Skrutskie M. F., 1989, AJ, 97, 1451

Torres C. A. O., Quast G. R., Melo C. H. F., Sterzik M. F., 2008, Handbook of Star Forming Regions, Volume II, 5, 757

Vacca W. D., Sandell G., 2011, ApJ, 732, 8

White R. J., Basri G., 2003, ApJ, 582, 1109

Williams J. P., Cieza L. A., 2011, ARA\&A, 49, 67

Wolf C. et al., 2018, Publ. Astron. Soc. Aust., 35, 10

York D. G. et al., 2000, AJ, 120, 1579

Zuckerman, B., Song, I., 2004, ARA\&A, 42, 685

Zuckerman, B., Song, I., 2012, ApJ, 758, 77

Zuckerman B., Vican L., Rodriguez D. R., 2014, ApJ, 788, 102

Zuckerman B., 2015, ApJ, 798, 86

Zuckerman B., 2019, ApJ, 870, 27

This paper has been typeset from a $\mathrm{TE}_{\mathrm{E}} \mathrm{X} / \mathrm{LAT} \mathrm{T}$ file prepared by the author. 